\title{
Protein-Remodeling Factors As Potential Therapeutics for Neurodegenerative Disease
}

\author{
Meredith E. Jackrel * and James Shorter* \\ Department of Biochemistry and Biophysics, Perelman School of Medicine at the University of Pennsylvania, Philadelphia, \\ PA, USA
}

\section{OPEN ACCESS}

Edited by:

Tiago Fleming Outeiro, University Medical Center Goettingen,

Germany

Reviewed by:

Luigi Bubacco,

University of Padua, Italy

Martin Lothar Duennwald, University of Western Ontario, Canada

Salvador Ventura,

Autonomous University of Barcelona

Spain

*Correspondence:

Meredith E. Jackrel

mjackre/@mail.med.upenn.edu James Shorter

jshorter@mail.med.upenn.edu

Specialty section: This article was submitted to

Neurodegeneration,

a section of the journal

Frontiers in Neuroscience

Received: 20 December 2016 Accepted: 15 February 2017 Published: 28 February 2017

Citation:

Jackrel ME and Shorter J (2017) Protein-Remodeling Factors As

Potential Therapeutics for

Neurodegenerative Disease.

Front. Neurosci. 11:99.

doi: 10.3389/fnins.2017.00099
Protein misfolding is implicated in numerous neurodegenerative disorders including amyotrophic lateral sclerosis, Parkinson's disease, Alzheimer's disease, and Huntington's disease. A unifying feature of patients with these disorders is the accumulation of deposits comprised of misfolded protein. Aberrant protein folding can cause toxicity through a loss or gain of protein function, or both. An intriguing therapeutic approach to counter these disorders is the application of protein-remodeling factors to resolve these misfolded conformers and return the proteins to their native fold and function. Here, we describe the application of protein-remodeling factors to alleviate protein misfolding in neurodegenerative disease. We focus on Hsp104, Hsp110/Hsp70/Hsp40, NMNAT, and HtrA1, which can prevent and reverse protein aggregation. While many of these protein-remodeling systems are highly promising, their activity can be limited. Thus, engineering protein-remodeling factors to enhance their activity could be therapeutically valuable. Indeed, engineered Hsp104 variants suppress neurodegeneration in animal models, which opens the way to novel therapeutics and mechanistic probes to help understand neurodegenerative disease.

Keywords: protein-remodeling factors, protein-misfolding disease, neurodegeneration, Hsp104, Hsp70, Hsp110, NMNAT, HtrA1

\section{INTRODUCTION}

There are numerous devastating, and incurable, neurodegenerative disorders that are increasing in prevalence as our population ages (Dobson, 2003; Forman et al., 2004; Morimoto, 2006). These disorders include: Alzheimer's disease (AD), Parkinson's disease (PD), amyotrophic lateral sclerosis (ALS), and frontotemporal dementia (FTD) (Dobson, 2003; Forman et al., 2004; Morimoto, 2006; Lagier-Tourenne et al., 2010; Robberecht and Philips, 2013). Treatments for these disorders remain palliative, and no therapeutics are available that address their underlying cause (Forman et al., 2004; Robberecht and Philips, 2013). Furthermore, each of these disorders manifests in different ways in patients. For instance, AD patients have impaired memory yet their movement is preserved, while ALS patients' memory is preserved while their control of movement becomes impaired (Forman et al., 2004; Lagier-Tourenne et al., 2010; Robberecht and Philips, 2013). Yet, at the fundamental level, these neurodegenerative disorders are linked by the presence of insoluble proteinaceous inclusions in the brain (Dobson, 2003; Forman et al., 2004; Lagier-Tourenne et al., 2010; Robberecht and Philips, 2013). 
It is important to note that these neurodegenerative diseases are not due to mass protein misfolding, but instead the misfolding of specific proteins are implicated in each disease (Dobson, 2003; Cushman et al., 2010). For instance, $\alpha$-synuclein misfolds into amyloid fibrils that accumulate in Lewy bodies in the dopamine neurons of PD patients, while in ALS patients TDP-43 or FUS misfold into cytoplasmic aggregates in degenerating motor neurons and glia (Spillantini et al., 1997; Neumann et al., 2006; Chen-Plotkin et al., 2010; Mackenzie et al., 2010; Robberecht and Philips, 2013; Dehay et al., 2015). These proteins, as well as many others that underpin diverse neurodegenerative disorders, are expressed in nearly all cells. Yet it remains perplexing what initiates and drives the misfolding of specific proteins in specific neuronal subtypes, leading to subtype-specific neurodegeneration (Saxena and Caroni, 2011). Additionally, it remains unclear if neuronal degeneration is always a direct consequence of aggregate accumulation. Indeed, many of these proteins serve essential functions, and so a loss of function due to aggregation could alternatively lead to toxicity (Winklhofer et al., 2008; Yang et al., 2014; O’Rourke et al., 2016).

In each of these neurodegenerative disorders, the protein homeostasis (proteostasis) network ultimately fails to combat the accumulation of misfolded conformers, consequently leading to disease (Balch et al., 2008; Shorter, 2016). To address the protein-misfolding problem, there are several avenues that could be explored. First, degradation of the toxic, misfolded conformers might be beneficial. For instance, in some PD patients, an increase in $\alpha$-synuclein levels is implicated, and thus degradation of this excess $\alpha$-synuclein might be beneficial (Ebrahimi-Fakhari et al., 2012). A similar strategy might be useful in Huntington's disease patients (Yamamoto et al., 2000). Alternatively, stalling the protein-misfolding process is an effective means of therapeutically treating patients with familial amyloid neuropathy (FAP) (Bulawa et al., 2012; Cho et al., 2015; Ankarcrona et al., 2016). FAP is caused by the misfolding of transthyretin, which forms amyloid fibrils that accumulate in various tissues and organs, ultimately leading to organ failure. To combat FAP, the drug Tafamidis was developed to stabilize the native tetrameric form of transthyretin, thus blocking further misfolding and stalling the amyloid cascade. Tafamidis is approved for use by the European Medicines Agency, and is the only therapeutic in use that mitigates neurodegenerative disease by preventing protein misfolding (Ruberg and Berk, 2012). Additionally the drug Tolcapone, which is FDA-approved for PD, was found to also stabilize transthyretin and block aggregation (Sant'Anna et al., 2016). A similar strategy to pharmacologically stabilize $\alpha$-crystallins may effectively block their misfolding and aggregation and treat cataracts (Makley et al., 2015). The success of Tafamidis provides strong proof of concept that targeting protein misfolding can be therapeutically effective (Bulawa et al., 2012; Cho et al., 2015; Ankarcrona et al., 2016). Additionally, clinical trials are ongoing to assess the efficacy of antibodies aimed at clearing plaques comprised of $\mathrm{A} \beta$ that accumulate in $\mathrm{AD}$ patients (Sevigny et al., 2016), though notably one trial recently failed. Indeed, an additional intriguing possibility would be to remodel the misfolded species such that the protein regains its functional, native conformation, which would simultaneously mitigate toxicity due to loss-of-function or gain-of-function (Jackrel and Shorter, 2014b, 2015; Mack and Shorter, 2016; Shorter, 2016). However, many of the proteins that misfold in these disorders adopt a cross-beta fibrillar form, termed amyloid, which is a highly stable and self-templating structure (Dobson, 2003). Nonetheless, protein-remodeling factors that have evolved to antagonize protein misfolding could be harnessed to reverse deleterious protein misfolding in disease (Table 1).

The proteostasis network ultimately collapses in neurodegenerative disease (Shorter, 2016). This network is comprised of many molecular chaperones that normally promote the proper folding of disease-associated proteins, as well as the entire proteome. Thus, an intriguing way to address the collapse of the proteostasis network would be to remedy or rewire this network (Jackrel et al., 2014a; Jackrel and Shorter, 2014b, 2015). This approach could be pursued by either enhancing and tuning the activity of endogenously expressed protein-remodeling factors, or by introducing new protein-remodeling factors that are not normally expressed (Warrick et al., 1999; Auluck et al., 2002; Jackrel et al., 2014a). Many protein-remodeling factors have been proposed to function in alleviating protein misfolding, including: Hsp104, Hsp110/Hsp70/Hsp40, NMNAT, and HtrA1 (Zhai et al., 2008; Jackrel and Shorter, 2014b, 2015; Poepsel et al., 2015; Ali et al., 2016; Mack and Shorter, 2016; Shorter, 2016). Some of these proteins are capable of actively disaggregating and restoring the solubility of the misfolded conformers (Warrick et al., 1999; Auluck et al., 2002; Jackrel and Shorter, 2014a; Jackrel et al., 2014a). Thus, the application of protein-remodeling factors in a therapeutic setting is a highly promising avenue to address neurodegenerative disease. In this review, we discuss the potential application of these molecular chaperones and protein disaggregases in the development of therapeutics for neurodegenerative disorders. These agents might be harnessed for therapeutic purposes through upregulation or through the introduction of exogenous protein through either gene therapy using adeno-associated viral vector technologies or direct injection. Alternatively, protein-remodeling factors could be therapeutically modulated using small molecules or even potentiated via engineering. We focus on efforts to reformulate a robust protein disaggregase from yeast, Hsp104, which has several unique properties that make it a particularly promising protein-remodeling factor for further exploration and application to reverse the protein misfolding implicated in numerous devastating neurodegenerative diseases (Lo Bianco et al., 2008; DeSantis et al., 2012; Cushman-Nick et al., 2013; Jackrel and Shorter, 2014a,b, 2015; Jackrel et al., 2014a). We also discuss several other protein-remodeling factors that have been recently assessed for their capacity to suppress or reverse protein misfolding connected to neurodegenerative disease.

\section{Hsp70 BLOCKS PROTEIN MISFOLDING}

One of the first molecular chaperones to be explored as a possible therapeutic for combating neurodegenerative disease was Hsp70. The Hsp70 family of proteins serves diverse functions in protein folding. Hsp70 promotes the refolding of aggregated or misfolded 
TABLE 1 | Protein-remodeling factors can remodel diverse substrates.

\begin{tabular}{|c|c|c|}
\hline Protein remodeling factor & Activity & Substrates remodeled \\
\hline Hsp70 & Blocks misfolding & Polyglutamine, $\alpha$-syn, $A \beta$ \\
\hline Hsp110/Hsp70/Hsp40 & Dissolves preformed aggregates & SOD1, $\alpha$-syn \\
\hline NMNAT & Dissolves preformed aggregates & Tau \\
\hline Htra1 & Dissolves and degrades preformed aggregates & $A \beta$ and tau \\
\hline Hsp104 & Dissolves preformed aggregates, amyloid, and pre-amyloid oligomers & $\alpha$-syn, TDP-43, FUS, A $\beta$, tau, polyglutamine \\
\hline
\end{tabular}

proteins (Mayer and Bukau, 2005; Mack and Shorter, 2016). It also serves to ensure the proper folding of newly synthesized proteins (Mayer and Bukau, 2005). To do so, Hsp70 functions cooperatively with its co-chaperone, Hsp40, to bind and thus protect hydrophobic stretches harbored by its clients (Mayer and Bukau, 2005; Mashaghi et al., 2016). This function is crucial during protein synthesis, but is also important following cellular stresses that partially denature mature proteins, because by binding exposed stretches on these partially denatured proteins, Hsp70 can block protein aggregation (Mayer and Bukau, 2005; Mack and Shorter, 2016). Thus, in disease, upregulation of Hsp70 might prevent protein aggregation and promote the restoration of proteostasis. A Drosophila model of polyglutamine misfolding has been established in which overexpression of polyglutamine leads to neurodegeneration (Warrick et al., 1998). In this model, overexpression of Hsp70 suppressed polyglutamineinduced neurodegeneration (Warrick et al., 1999). Similarly, in a Drosophila model of $\alpha$-synuclein misfolding, Hsp70 suppressed neurodegeneration (Auluck et al., 2002). However, it is important to note that while Hsp70 inhibited neurodegeneration in these models, it was not found to solubilize aggregates (Warrick et al., 1999; Auluck et al., 2002; Cushman-Nick et al., 2013). Nonetheless, in a mouse model of ALS, intraperitoneal injection of human Hsp70 increased lifespan, delayed the onset of symptoms, arrested denervation, preserved axonal function, and prolonged motor neuron viability (Gifondorwa et al., 2007, 2012).

Elevating Hsp70 expression can slow neurodegeneration in fly and mouse models (Warrick et al., 1999; Auluck et al., 2002; Gifondorwa et al., 2007, 2012). Hsp70 likely becomes overwhelmed in neurodegenerative disease. Thus, it may be important to enhance Hsp70 activity via potentiating mutations or small molecules (Mack and Shorter, 2016; Shorter, 2016). Indeed, using protein-engineering techniques the activity of the bacterial homolog of Hsp70, DnaK, has been enhanced and these variants demonstrate elevated luciferase refolding activity (Aponte et al., 2010; Schweizer et al., 2011). Recently, Hsp70 engineering has been extended to human Hsp70 and neurodegenerative disease-associated substrates (Aprile et al., 2015). Here, Hsp70 was tuned through rational design to more potently bind $\alpha$-synuclein and A $\beta 42$. Peptides complementary to target epitopes in $\alpha$-synuclein and $\mathrm{A} \beta 42$ were developed, and these peptides were introduced into the C-terminal region of Hsp70 (Aprile et al., 2015). While introduction of these peptides enhanced the binding affinity of Hsp70 to $\alpha$-synuclein and A $\beta 42$, binding to other client proteins was unaffected (Aprile et al.,
2015). Thus, tuning Hsp70 to broaden its substrate specificity does not come at the cost of restricted capacity to regulate its diverse client pool (Aprile et al., 2015). Additionally, small molecules have been identified that can enhance specific aspects of Hsp70 activity. For instance, four small molecules: MKT-077, JG-98, YM-1, and YM-8 bind the nucleotide-binding domain of Hsp70 in the ADP, but not ATP-bound state. This binding stabilizes the ADP-bound state resulting in increased affinity of Hsp70 for its clients, which can under some circumstances lead to their enhanced folding (Rousaki et al., 2011; Miyata et al., 2013; Wang et al., 2013; Shorter, 2016). In the cellular environment, YM-1 promotes clearance of polyglutamine oligomers and aggregates (Wang et al., 2013). All four of these molecules promote the clearance of tau and are therapeutically beneficial in tauopathy models (Abisambra et al., 2013; Miyata et al., 2013; Fontaine et al., 2015).

\section{THE METAZOAN PROTEIN-DISAGGREGASE SYSTEM: Hsp110/Hsp70/Hsp40}

It has long been hypothesized that humans might possess a protein disaggregase similar to those in the Hsp100 family of proteins that are highly conserved in bacteria, fungi, and plants (Shorter, 2008, 2011; Torrente and Shorter, 2013). However, the discovery of such a protein disaggregase has been elusive until it was discovered that Hsp110 in collaboration with Hsp70 and Hsp40 can disaggregate and reactivate protein (Shorter, 2011; Mattoo et al., 2013; Torrente and Shorter, 2013; Finka et al., 2015; Gao et al., 2015; Nillegoda and Bukau, 2015; Nillegoda et al., 2015). Hsp110 is an Hsp70 family member that in collaboration with Hsp70 and Hsp40 can disaggregate preformed aggregates and amyloid (Shorter, 2011; Duennwald et al., 2012; Gao et al., 2015; Nillegoda et al., 2015). Hsp110 collaborates and synergizes with Hsp70 and two classes of Hsp40 cochaperones to resolve large protein aggregates (Nillegoda and Bukau, 2015; Nillegoda et al., 2015). It is hypothesized that due to the large number of possible complexes that could form between different Hsp70s and Hsp40s, distinct and specific complexes might be harnessed to dissolve different protein aggregates (Nillegoda and Bukau, 2015; Nillegoda et al., 2015). Perhaps one specific combination might be employed in specific neuronal subtypes, or a given combination might specifically disaggregate $\alpha$-synuclein while another might specifically disaggregate tau. 
Ultimately, failure of the Hsp110/Hsp70/Hsp40 system might underpin numerous protein-misfolding disorders, and restoration or specific activation of this system might be therapeutically useful (Nillegoda and Bukau, 2015; Shorter, 2016). Indeed, overexpression of Hsp110 with Hsp40 suppressed the toxicity induced by polyglutamine overexpression in Drosophila, though it is not apparent if Hsp110 modulates polyglutamine aggregation (Kuo et al., 2013). Additionally, transgenic overexpression of Hsp110 in neurons enhanced survival in ALS model mice, but again, the effects of Hsp110 on SOD1 aggregation were not assessed in these experiments (Nagy et al., 2016). It remains unclear if upregulation of Hsp110 levels will be sufficient to restore normal functionality in animal models, and ultimately in humans. It may be useful to tune the activity of the Hsp110/Hsp70/Hsp40 system using protein-engineering techniques, or alternatively, small-molecule modulators could be developed to enhance the activity of this system. Small heat-shock proteins can also enhance the disaggregase activity of this system (Duennwald et al., 2012), and might also be targeted therapeutically (Makley et al., 2015). However, determining precisely how to therapeutically boost the activity of this system comprised of several components may prove challenging.

\section{NMNAT}

Nicotinamide mononucleotide adenylyl transferases (NMNATs) are nicotinamide adenine dinucleotide (NAD)-synthesizing enzymes. NAD is an important cofactor that mediates numerous cellular processes. NMNATs are important in neuronal maintenance, thus NMNAT knockdown leads to axonal degeneration, while NMNAT overexpression is neuroprotective in several animal models of neurodegeneration (Zhai et al., 2008; Gilley and Coleman, 2010; Ali et al., 2016). NMNAT2 is highly expressed in the mammalian brain, and NMNAT2 mRNA levels are reduced in $\mathrm{PD}, \mathrm{HD}, \mathrm{AD}$, and tauopathy patients (Ali et al., 2016). Furthermore, elevating NMNAT2 levels in tauopathy model mice suppressed neurodegeneration (Ljungberg et al., 2012). Additionally, NMNAT2 mRNA levels correlate positively with cognitive function and negatively with the pathological features of AD (Ali et al., 2016). In AD brains, NMNAT2 mRNA and protein levels are greatly reduced relative to controls, and NMNAT2 co-localizes with aggregated tau (Ali et al., 2016). NMNAT2 overexpression can reduce the pathological accumulation of hyperphosphorylated tau without altering total tau levels (Ljungberg et al., 2012; Ali et al., 2016). NMNAT2 can prevent protein denaturation and promote protein refolding with similar activity to Hsp70 (Ali et al., 2016). Surprisingly, this activity is maintained even in enzymatically-dead NMNAT2 mutants that lack NAD synthetic activity (Ali et al., 2016). These enzymatically-dead NMNAT2 mutants also reduced hyperphosphorylated tau levels (Ali et al., 2016).

NMNAT2 has been demonstrated to form a complex with Hsp90 to solubilize and refold aggregated substrates (Ali et al., 2016). Moreover, deletion of NMNAT2 increases the vulnerability of cortical neurons to proteotoxic stress (Ali et al., 2016). Thus, therapeutically upregulating NMNAT or enhancing NMNAT activity via small-molecule modulation might be effective in regulating tau levels. It will be interesting to assess the protein-remodeling activity of NMNATs against the many other substrates implicated in protein-misfolding disorders. Given the fundamental role NMNATs play in neuronal maintenance, failure of NMNATs to combat protein misfolding might be common to many other disease-associated substrates in addition to tau.

\section{HtrA1 CAN DISAGGREGATE AND DEGRADE TOXIC CONFORMERS}

HtrAl is a PDZ serine protease that disassembles tau and $A \beta$ fibrils, which are linked to $\mathrm{AD}$, and then degrades them (Poepsel et al., 2015). Intriguingly, HtrA1 is found in the cytoplasm and is also secreted (Poepsel et al., 2015). Correlating with this pattern, $A \beta 42$ fibrils are found in the extracellular space while tau fibrils are found in the cytoplasm. Thus, it has been hypothesized that HtrAl might be a system that naturally disaggregates and degrades A $\beta 42$ and tau (Poepsel et al., 2015; Shorter, 2016). Indeed, HtrA1 activity might be insufficient in $\mathrm{AD}$ patients (Shorter, 2016). Therefore, boosting and finetuning the activity of HtrAl might be valuable in combating AD. It has been demonstrated that HtrA1 activity can be tuned through protein engineering. The disassembly and degradation activities of HtrAl can be separated, as protease-defective HtrA1 variants dissolve but do not degrade $A \beta$ fibrils, providing HtrA1 variants that can either dissolve the aggregates or dissolve and degrade the aggregates (Poepsel et al., 2015). The ability to separate or combine disassembly and degradation activities in a single protein is very valuable, and might find utility in certain situations. Therefore, it will be very interesting to engineer substrate-specific HtrA1 variants that can target substrates beyond $\mathrm{A} \beta$ and tau. These substrate-specific variants could be constructed in both the disaggregate-only or disaggregateand-degrade backgrounds. This advance would allow for the flexibility to reactivate proteins that serve beneficial functions. Alternatively, subsets of PD patients show increased $\alpha$-synuclein levels (Ebrahimi-Fakhari et al., 2012), and thus for these patients it may be beneficial to not just solubilize $\alpha$-synuclein, but also to degrade it. Additionally, small-molecule enhancers of HtrA1 have been identified, and so it will be important to test the effects of these compounds in various models of protein-misfolding disorders (Jo et al., 2014). It will also be important to assess if HtrA1 can clear highly toxic pre-amyloid oligomeric forms of $A \beta$ and tau, or only the fibrils.

\section{Hsp104 VARIANTS SUPPRESS PROTEIN MISFOLDING, MISLOCALIZATION, AND TOXICITY IN YEAST AND ANIMAL MODELS}

Hsp104 is a ring-shaped hexameric AAA + protein from yeast that serves two distinct functions (Sweeny and Shorter, 2016; Yokom et al., 2016). First, it solubilizes proteins that aggregate 
following cellular stress to promote yeast survival (Parsell et al., 1991, 1994; Glover and Lindquist, 1998; Glover and Tkach, 2001; Wallace et al., 2015). Second, it regulates yeast prion formation and dissolution (Chernoff et al., 1995; Shorter and Lindquist, 2004, 2005, 2006; Sweeny and Shorter, 2008, 2016; Sweeny et al., 2015). In serving these two roles, Hsp104 recognizes and regulates a diverse milieu of substrates, comprised of the entire yeast proteome, as well as yeast prions (Newby and Lindquist, 2013). While Hsp104 is highly conserved in bacteria, fungi, and plants, Hsp104 has no metazoan homolog (Erives and Fassler, 2015).

The amyloid fold is a highly conserved protein structure, thus it was hypothesized that the natural capacity of Hsp104 to recognize and solubilize yeast prions might translate to a capacity to recognize and solubilize diverse amyloid species associated with human disease (DeSantis et al., 2012; Jackrel and Shorter, 2014b, 2015; Jackrel et al., 2014a). Indeed, using purified proteins, Hsp104 has been shown to solubilize diverse amyloid species implicated in human disease including: $A \beta, \alpha-$ synuclein, polyglutamine expansions, prion protein, tau, and amylin (Liu et al., 2011; DeSantis et al., 2012; Jackrel and Shorter, 2014a; Jackrel et al., 2014a). Additionally, Hsp104 suppresses proteotoxicity in animal models (Satyal et al., 2000; Vacher et al., 2005; Lo Bianco et al., 2008; Cushman-Nick et al., 2013; Jackrel et al., 2014a). In a transgenic mouse model of HD, Hsp104 extended lifespan and decreased aggregate load (Vacher et al., 2005). Furthermore, Hsp104 has been demonstrated to be neuroprotective in a rat model of PD (Lo Bianco et al., 2008). Here, lentiviral vectors coding for $\alpha$-synuclein were injected into the substantia nigra of rats, and following 6 weeks of expression, brain slices were stained for dopaminergic markers. In this system, Hsp104 co-expression was neuroprotective and no offtarget effects were observed (Lo Bianco et al., 2008). Additionally, Hsp104 has been shown to directly clear preformed oligomeric forms of $\alpha$-synuclein as well as eliminate self-templating $\alpha$ synuclein conformers. These experiments have provided strong evidence that Hsp104 may have therapeutic value. However, the activity of Hsp104 in suppressing degeneration in these animal models is limited as complete neuroprotection is not achieved (Vacher et al., 2005; Lo Bianco et al., 2008).

We have enhanced the activity of Hsp104 via engineering (Jackrel et al., 2014a,b, 2015; Jackrel and Shorter, 2014a). We have constructed large libraries of randomized Hsp104 variants and developed screening techniques to isolate enhanced variants. When overexpressed in yeast, the proteins TDP-43, FUS, and $\alpha$-synuclein all form cytoplasmic foci and are toxic (Outeiro and Lindquist, 2003; Johnson et al., 2008; Sun et al., 2011). These yeast models have also empowered the identification of genetic risk factors for these disorders (Elden et al., 2010; Ju et al., 2011; Sun et al., 2011). Deletion or overexpression of Hsp104 does not suppress the toxicity or aggregation of these proteins in yeast (Jackrel et al., 2014a). Thus, these yeast models provide an ideal screening platform to isolate Hsp104 variants with a gain of therapeutic function (Jackrel et al., 2014a,b, 2015). Using these yeast assays, we have identified numerous Hsp104 variants that potently suppress TDP-43, FUS, and $\alpha$-synuclein toxicity (Jackrel and Shorter, 2014a,b, 2015;
Jackrel et al., 2014a,b, 2015). In addition to their suppression of toxicity, these variants also dissolved cytoplasmic foci of TDP-43, FUS, and $\alpha$-synuclein (Jackrel and Shorter, 2014a; Jackrel et al., 2014a, 2015). Furthermore, the potentiated variants restored alpha-synuclein to the plasma membrance and TDP43 to the nucleus (Jackrel et al., 2014a). These results are very promising. TDP-43 must shuttle to the nucleus to fulfill its roles in RNA homeostasis, and restoration of nuclear TDP-43 suggests that solubilization of TDP-43 can restore natively folded and functional TDP-43 (Jackrel et al., 2014a). These potentiated Hsp104 variants clear preformed TDP-43, FUS, and $\alpha$-synuclein fibrils at concentrations where Hsp104 is ineffective (Jackrel et al., 2014a).

To assess the therapeutic utility of potentiated Hsp104 variants, they have been tested in a C. elegans model of PD (Jackrel et al., 2014a). Here, the potentiated Hsp104 variants were robustly neuroprotective, while Hsp104 and an ATPase-dead negative control showed no activity (Jackrel et al., 2014a). To further demonstrate the therapeutic possibilities of potentiated Hsp104 variants, it will be essential to demonstrate their activity in additional neuronal models including mammalian neurons. It will also be important to develop additional potentiated Hsp104 variants with improved properties. To do so, it will be crucial to apply additional protein engineering techniques to enhance the activity and substrate specificity of the potentiated variants. There are numerous examples of proteins that are believed to have evolved from roles as generalists to specialists. Thus, it will be very interesting to see if laboratory techniques can accelerate this process for Hsp104 and produce finely-tuned variants. Additionally, perhaps Hsp104 variants can be produced to target pre-amyloid oligomers vs. fibrils and vice versa. In addition to being potentially of direct therapeutic benefit, these variants may hold great value in unraveling the key contributors and drivers of neurodegenerative disease. For instance, engineered disaggregases that can solubilize and reactivate oligomers but not fibrils may be employed as precise mechanistic probes to investigate the effects of resolving specific protein species. Also, the discovery that very subtle modification of natural protein-remodeling factors can confer dramatic alterations in chaperone activity (Jackrel et al., 2014a, 2015) suggests that subtle modification of other protein-remodeling factors, including Hsp110/Hsp70/Hsp40, NMNAT, and HtrA1 might also be amenable to potentiation.

\section{CONCLUSIONS AND FUTURE DIRECTIONS}

Protein misfolding is an enormously challenging issue that underpins many of the most devastating diseases facing society. As the population continues to age, the toll of neurodegenerative disease will continue to rise. Unfortunately, while substantial efforts have been mounted to counter these disorders, there are no treatments available for any of these diseases (with the exception of Tafamidis for FAP). Thus, in the development of new therapeutics to combat these disorders, it will be important to employ innovative approaches. While it is unknown 
what specifically causes proteins to misfold and cause disease, the accumulation of misfolded aggregates, amyloid, and preamyloid species are key contributors to pathogenesis. Therefore, if protein-misfolding trajectories could be reversed, perhaps so could these diseases. Protein-remodeling factors, which have the capacity to block and even reverse protein misfolding might be uniquely positioned as potential therapeutics. Many proteinremodeling factors have been assessed and demonstrated to be potentially useful in combating these disorders. For instance, increased levels or activity of the protein HtrA1 might be employed to dissolve and degrade both tau and $\mathrm{A} \beta$ aggregates in $\mathrm{AD}$ patients. However, as HtrA1, as well as NMNAT and Hsp110/Hsp70/Hsp40, are all present in humans, it appears that these systems are either insufficient to prevent pathogenesis or are compromised in certain individuals. Thus, it will be important to continue to focus not just on the application of these chaperones directly in disease models, but also to continue to develop approaches to boost and nuance these proteinremodeling systems.

While highly promising, the idea of modulating the proteostasis network is not without caveats. For instance, upregulation of protein-remodeling factors might be beneficial to enhance protein folding and combat neurodegenerative disorders, yet enhanced protein folding might also enable cell proliferation which could promote cancers. Nonetheless, protein-remodeling factors present a unique opportunity to restore proteins to their native fold and function, thus simultaneously alleviating both a loss or gain of function. As with all therapeutics, it will be important to assess for

\section{REFERENCES}

Abisambra, J., Jinwal, U. K., Miyata, Y., Rogers, J., Blair, L., Li, X., et al. (2013). Allosteric heat shock protein 70 inhibitors rapidly rescue synaptic plasticity deficits by reducing aberrant tau. Biol. Psychiatry 74, 367-374. doi: 10.1016/j.biopsych.2013.02.027

Ali, Y. O., Allen, H. M., Yu, L., Li-Kroeger, D., Bakhshizadehmahmoudi, D., Hatcher, A., et al. (2016). NMNAT2:HSP90 complex mediates proteostasis in proteinopathies. PLoS Biol. 14:e1002472. doi: 10.1371/journal.pbio.10 02472

Ankarcrona, M., Winblad, B., Monteiro, C., Fearns, C., Powers, E. T., Johansson, J., et al. (2016). Current and future treatment of amyloid diseases. J. Intern. Med. 280, 177-202. doi: 10.1111/joim.12506

Aponte, R. A., Zimmermann, S., and Reinstein, J. (2010). Directed evolution of the DnaK chaperone: mutations in the lid domain result in enhanced chaperone activity. J. Mol. Biol. 399, 154-167. doi: 10.1016/j.jmb.2010.03.060

Aprile, F. A., Sormanni, P., and Vendruscolo, M. (2015). A rational design strategy for the selective activity enhancement of a molecular chaperone toward a target substrate. Biochemistry 54, 5103-5112. doi: 10.1021/acs.biochem.5b00459

Auluck, P. K., Chan, H. Y., Trojanowski, J. Q., Lee, V. M., and Bonini, N. M. (2002). Chaperone suppression of alpha-synuclein toxicity in a Drosophila model for Parkinson's disease. Science 295, 865-868. doi: 10.1126/science.1067389

Balch, W. E., Morimoto, R. I., Dillin, A., and Kelly, J. W. (2008). Adapting proteostasis for disease intervention. Science 319, 916-919. doi: 10.1126/science.1141448

Bulawa, C. E., Connelly, S., Devit, M., Wang, L., Weigel, C., Fleming, J. A., et al. (2012). Tafamidis, a potent and selective transthyretin kinetic stabilizer that inhibits the amyloid cascade. Proc. Natl. Acad. Sci. U.S.A. 109, 9629-9634. doi: 10.1073/pnas.1121005109 possible off-target effects. For instance, in developing new disaggregase technologies, it will be important to harness protein disaggregation to avoid the unfolding of functional protein complexes. However, it is important to note that Hsp104 does not unfold natively folded proteins. Regardless, it will be important to continue to engineer protein-remodeling factors with desired traits, such as enhanced substrate specificity. New approaches to develop small-molecule modulators of protein-remodeling systems, as well as the engineering of tailored protein-remodeling systems, will prove invaluable in our efforts to rewire and restore the proteostasis network and thus combat neurodegenerative disease.

\section{AUTHOR CONTRIBUTIONS}

MJ and JS wrote and revised the review.

\section{FUNDING}

MJ is supported by a Target ALS Springboard Fellowship and American Heart Association Postdoctoral Fellowship. JS is supported by the NIH (R01GM099836 and R21NS090205), ALS Association, and the Robert Packard Center for ALS Research at Johns Hopkins.

\section{ACKNOWLEDGMENTS}

We thank Edward Chuang, Korrie Mack, and Zachary March for feedback on the manuscript.

Chen-Plotkin, A. S., Lee, V. M., and Trojanowski, J. Q. (2010). TAR DNAbinding protein 43 in neurodegenerative disease. Nat. Rev. Neurol. 6, 211-220. doi: 10.1038/nrneurol.2010.18

Chernoff, Y. O., Lindquist, S. L., Ono, B., Inge-Vechtomov, S. G., and Liebman, S. W. (1995). Role of the chaperone protein Hsp104 in propagation of the yeast prion-like factor [psi+]. Science 268, 880-884.

Cho, Y., Baranczak, A., Helmke, S., Teruya, S., Horn, E. M., Maurer, M. S., et al. (2015). Personalized medicine approach for optimizing the dose of tafamidis to potentially ameliorate wild-type transthyretin amyloidosis (cardiomyopathy). Amyloid 22, 175-180. doi: 10.3109/13506129.2015.1063485

Cushman, M., Johnson, B. S., King, O. D., Gitler, A. D., and Shorter, J. (2010). Prion-like disorders: blurring the divide between transmissibility and infectivity. J. Cell Sci. 123(Pt 8), 1191-1201. doi: 10.1242/jcs.051672

Cushman-Nick, M., Bonini, N. M., and Shorter, J. (2013). Hsp104 suppresses polyglutamine-induced degeneration post onset in a drosophila MJD/SCA3 model. PLoS Genet. 9:e1003781. doi: 10.1371/journal.pgen.1003781

Dehay, B., Bourdenx, M., Gorry, P., Przedborski, S., Vila, M., Hunot, S., et al. (2015). Targeting alpha-synuclein for treatment of Parkinson's disease: mechanistic and therapeutic considerations. Lancet Neurol. 14, 855-866. doi: 10.1016/S1474-4422(15)00006-X

DeSantis, M. E., Leung, E. H., Sweeny, E. A., Jackrel, M. E., Cushman-Nick, M., Neuhaus-Follini, A., et al. (2012). Operational plasticity enables hsp104 to disaggregate diverse amyloid and nonamyloid clients. Cell 151, 778-793. doi: 10.1016/j.cell.2012.09.038

Dobson, C. M. (2003). Protein folding and misfolding. Nature 426, 884-890. doi: $10.1038 /$ nature 02261

Duennwald, M. L., Echeverria, A., and Shorter, J. (2012). Small heat shock proteins potentiate amyloid dissolution by protein disaggregases from yeast and humans. PLoS Biol. 10:e1001346. doi: 10.1371/journal.pbio.1001346 
Ebrahimi-Fakhari, D., McLean, P. J., and Unni, V. K. (2012). Alphasynuclein's degradation in vivo: opening a new (cranial) window on the roles of degradation pathways in Parkinson disease. Autophagy 8, 281-283. doi: 10.4161/auto.8.2.18938

Elden, A. C., Kim, H. J., Hart, M. P., Chen-Plotkin, A. S., Johnson, B. S., Fang, X., et al. (2010). Ataxin-2 intermediate-length polyglutamine expansions are associated with increased risk for ALS. Nature 466, 1069-1075. doi: 10.1038 /nature09320

Erives, A. J., and Fassler, J. S. (2015). Metabolic and chaperone gene loss marks the origin of animals: evidence for Hsp104 and Hsp78 chaperones sharing mitochondrial enzymes as clients. PLoS ONE 10:e117192. doi: 10.1371/journal.pone.0117192

Finka, A., Sharma, S. K., and Goloubinoff, P. (2015). Multi-layered molecular mechanisms of polypeptide holding, unfolding and disaggregation by HSP70/HSP110 chaperones. Front. Mol. Biosci. 2:29. doi: $10.3389 /$ fmolb.2015.00029

Fontaine, S. N., Martin, M. D., Akoury, E., Assimon, V. A., Borysov, S., Nordhues, B. A., et al. (2015). The active Hsc70/tau complex can be exploited to enhance tau turnover without damaging microtubule dynamics. Hum. Mol. Genet. 24, 3971-3981. doi: 10.1093/hmg/ddv135

Forman, M. S., Trojanowski, J. Q., and Lee, V. M. (2004). Neurodegenerative diseases: a decade of discoveries paves the way for therapeutic breakthroughs. Nat. Med. 10, 1055-1063. doi: 10.1038/nm1113

Gao, X., Carroni, M., Nussbaum-Krammer, C., Mogk, A., Nillegoda, N. B., Szlachcic, A., et al. (2015). Human Hsp70 disaggregase reverses Parkinson's-linked alpha-synuclein amyloid fibrils. Mol. Cell 59, 781-793. doi: 10.1016/j.molcel.2015.07.012

Gifondorwa, D. J., Jimenz-Moreno, R., Hayes, C. D., Rouhani, H., Robinson, M. B., Strupe, J. L., et al. (2012). Administration of recombinant heat shock protein 70 delays peripheral muscle denervation in the SOD1(G93A) mouse model of amyotrophic lateral sclerosis. Neurol. Res. Int. 2012:170426. doi: 10.1155/2012/170426

Gifondorwa, D. J., Robinson, M. B., Hayes, C. D., Taylor, A. R., Prevette, D. M., Oppenheim, R. W., et al. (2007). Exogenous delivery of heat shock protein 70 increases lifespan in a mouse model of amyotrophic lateral sclerosis. J. Neurosci. 27, 13173-13180. doi: 10.1523/JNEUROSCI.4057-07.2007

Gilley, J., and Coleman, M. P. (2010). Endogenous Nmnat2 is an essential survival factor for maintenance of healthy axons. PLoS Biol. 8:e1000300. doi: 10.1371/journal.pbio.1000300

Glover, J. R., and Lindquist, S. (1998). Hsp104, Hsp70, and Hsp40: a novel chaperone system that rescues previously aggregated proteins. Cell 94, 73-82.

Glover, J. R., and Tkach, J. M. (2001). Crowbars and ratchets: hsp100 chaperones as tools in reversing protein aggregation. Biochem. Cell Biol. 79, 557-568. doi: 10.1139/bcb-79-5-557

Jackrel, M. E., and Shorter, J. (2014a). Potentiated Hsp104 variants suppress toxicity of diverse neurodegenerative disease-linked proteins. Dis. Model. Mech. 7, 1175-1184. doi: 10.1242/dmm.016113

Jackrel, M. E., and Shorter, J. (2014b). Reversing deleterious protein aggregation with re-engineered protein disaggregases. Cell Cycle 13, 1379-1383. doi: $10.4161 /$ cc.28709

Jackrel, M. E., and Shorter, J. (2015). Engineering enhanced protein disaggregases for neurodegenerative disease. Prion 9, 90-109. doi: 10.1080/19336896.2015.1020277

Jackrel, M. E., DeSantis, M. E., Martinez, B. A., Castellano, L. M., Stewart, R. M., Caldwell, K. A., et al. (2014a). Potentiated Hsp104 Variants antagonize diverse proteotoxic misfolding events. Cell 156, 170-182. doi: 10.1016/j.cell.2013.11.047

Jackrel, M. E., Tariq, A., Yee, K., Weitzman, R., and Shorter, J. (2014b). Isolating potentiated Hsp104 variants using yeast proteinopathy models. J. Vis. Exp. 93:e52089. doi: 10.3791/52089

Jackrel, M. E., Yee, K., Tariq, A., Chen, A. I., and Shorter, J. (2015). Disparate mutations confer therapeutic gain of Hsp104 function. ACS Chem. Biol. 10, 2672-2679. doi: 10.1021/acschembio.5b00765

Jo, H., Patterson, V., Stoessel, S., Kuan, C. Y., and Hoh, J. (2014). Protoporphyrins enhance oligomerization and enzymatic activity of HtrA1 serine protease. PLoS ONE 9:e115362. doi: 10.1371/journal.pone.0115362

Johnson, B. S., McCaffery, J. M., Lindquist, S., and Gitler, A. D. (2008). A yeast TDP-43 proteinopathy model: exploring the molecular determinants of
TDP-43 aggregation and cellular toxicity. Proc. Natl. Acad. Sci. U.S.A. 105, 6439-6444. doi: 10.1073/pnas.0802082105

Ju, S., Tardiff, D. F., Han, H., Divya, K., Zhong, Q., Maquat, L. E., et al. (2011). A yeast model of FUS/TLS-dependent cytotoxicity. PLoS Biol. 9:e1001052. doi: 10.1371/journal.pbio.1001052

Kuo, Y., Ren, S., Lao, U., Edgar, B. A., and Wang, T. (2013). Suppression of polyglutamine protein toxicity by co-expression of a heat-shock protein 40 and a heat-shock protein 110. Cell Death Dis. 4, e833. doi: 10.1038/cddis.2013.351

Lagier-Tourenne, C., Polymenidou, M., and Cleveland, D. W. (2010). TDP-43 and FUS/TLS: emerging roles in RNA processing and neurodegeneration. Hum. Mol. Genet. 19, R46-R64. doi: 10.1093/hmg/ddq137

Liu, Y. H., Han, Y. L., Song, J., Wang, Y., Jing, Y. Y., Shi, Q., et al. (2011). Heat shock protein 104 inhibited the fibrillization of prion peptide 106-126 and disassembled prion peptide 106-126 fibrils in vitro. Int. J. Biochem. Cell Biol. 43, 768-774. doi: 10.1016/j.biocel.2011.01.022

Ljungberg, M. C., Ali, Y. O., Zhu, J., Wu, C. S., Oka, K., Zhai, R. G., et al. (2012). CREB-activity and nmnat2 transcription are down-regulated prior to neurodegeneration, while NMNAT2 over-expression is neuroprotective, in a mouse model of human tauopathy. Hum. Mol. Genet. 21, 251-267. doi: $10.1093 / \mathrm{hmg} / \mathrm{ddr} 492$

Lo Bianco, C., Shorter, J., Regulier, E., Lashuel, H., Iwatsubo, T., Lindquist, S., et al. (2008). Hsp104 antagonizes alpha-synuclein aggregation and reduces dopaminergic degeneration in a rat model of Parkinson disease. J. Clin. Invest. 118, 3087-3097. doi: 10.1172/JCI35781

Mack, K. L., and Shorter, J. (2016). Engineering and evolution of molecular chaperones and protein disaggregases with enhanced activity. Front. Mol. Biosci. 3:8. doi: 10.3389/fmolb.2016.00008

Mackenzie, I. R., Rademakers, R., and Neumann, M. (2010). TDP-43 and FUS in amyotrophic lateral sclerosis and frontotemporal dementia. Lancet Neurol. 9, 995-1007. doi: 10.1016/S1474-4422(10)70195-2

Makley, L. N., McMenimen, K. A., DeVree, B. T., Goldman, J. W., McGlasson, B. N., Rajagopal, P., et al. (2015). Pharmacological chaperone for alpha-crystallin partially restores transparency in cataract models. Science 350, 674-677. doi: 10.1126/science.aac9145

Mashaghi, A., Bezrukavnikov, S., Minde, D. P., Wentink, A. S., Kityk, R., Zachmann-Brand, B., et al. (2016). Alternative modes of client binding enable functional plasticity of Hsp70. Nature 539, 448-451. doi: 10.1038/nature20137

Mattoo, R. U., Sharma, S. K., Priya, S., Finka, A., and Goloubinoff, P. (2013). Hsp110 is a bona fide chaperone using ATP to unfold stable misfolded polypeptides and reciprocally collaborate with Hsp70 to solubilize protein aggregates. J. Biol. Chem. 288, 21399-21411. doi: 10.1074/jbc.M113.479253

Mayer, M. P., and Bukau, B. (2005). Hsp70 chaperones: cellular functions and molecular mechanism. Cell. Mol. Life Sci. 62, 670-684. doi: 10.1007/s00018-004-4464-6

Miyata, Y., Li, X., Lee, H. F., Jinwal, U. K., Srinivasan, S. R., Seguin, S. P., et al. (2013). Synthesis and initial evaluation of YM-08, a blood-brain barrier permeable derivative of the heat shock protein 70 (Hsp70) inhibitor MKT-077, which reduces tau levels. ACS Chem. Neurosci. 4, 930-939. doi: $10.1021 / \mathrm{cn} 300210 \mathrm{~g}$

Morimoto, R. I. (2006). Stress, aging, and neurodegenerative disease. N. Engl. J. Med. 355, 2254-2255. doi: 10.1056/NEJMcibr065573

Nagy, M., Fenton, W. A., Li, D., Furtak, K., and Horwich, A. L. (2016). Extended survival of misfolded G85R SOD1-linked ALS mice by transgenic expression of chaperone Hsp110. Proc. Natl. Acad. Sci. U.S.A. 113, 5424-5428. doi: $10.1073 /$ pnas. 1604885113

Neumann, M., Sampathu, D. M., Kwong, L. K., Truax, A. C., Micsenyi, M. C., Chou, T. T., et al. (2006). Ubiquitinated TDP-43 in frontotemporal lobar degeneration and amyotrophic lateral sclerosis. Science 314, 130-133. doi: $10.1126 /$ science. 1134108

Newby, G. A., and Lindquist, S. (2013). Blessings in disguise: biological benefits of prion-like mechanisms. Trends Cell Biol. 23, 251-259. doi: 10.1016/j.tcb.2013.01.007

Nillegoda, N. B., and Bukau, B. (2015). Metazoan Hsp70-based protein disaggregases: emergence and mechanisms. Front. Mol. Biosci. 2:57. doi: $10.3389 / \mathrm{fmolb} .2015 .00057$

Nillegoda, N. B., Kirstein, J., Szlachcic, A., Berynskyy, M., Stank, A., Stengel, F., et al. (2015). Crucial HSP70 co-chaperone complex unlocks metazoan protein disaggregation. Nature 524, 247-251. doi: 10.1038/nature14884 
O’Rourke, J. G., Bogdanik, L., Yáñez, A., Lall, D., Wolf, A. J., Muhammad, A. K. M. G., et al. (2016). C9orf72 is required for proper macrophage and microglial function in mice. Science 351, 1324-1329. doi: 10.1126/science.aaf1064

Outeiro, T. F., and Lindquist, S. (2003). Yeast cells provide insight into alpha-synuclein biology and pathobiology. Science 302, 1772-1775. doi: 10.1126/science.1090439

Parsell, D. A., Kowal, A. S., Singer, M. A., and Lindquist, S. (1994). Protein disaggregation mediated by heat-shock protein Hsp104. Nature 372, 475-478. doi: $10.1038 / 372475 \mathrm{a} 0$

Parsell, D. A., Sanchez, Y., Stitzel, J. D., and Lindquist, S. (1991). Hsp104 is a highly conserved protein with two essential nucleotide-binding sites. Nature 353, 270-273. doi: 10.1038/353270a0

Poepsel, S., Sprengel, A., Sacca, B., Kaschani, F., Kaiser, M., Gatsogiannis, C., et al. (2015). Determinants of amyloid fibril degradation by the PDZ protease HTRA1. Nat. Chem. Biol. 11, 862-869. doi: 10.1038/nchembio.1931

Robberecht, W., and Philips, T. (2013). The changing scene of amyotrophic lateral sclerosis. Nat. Rev. Neurosci. 14, 248-264. doi: 10.1038/nrn3430

Rousaki, A., Miyata, Y., Jinwal, U. K., Dickey, C. A., Gestwicki, J. E., and Zuiderweg, E. R. (2011). Allosteric drugs: the interaction of antitumor compound MKT-077 with human Hsp70 chaperones. J. Mol. Biol. 411, 614-632. doi: 10.1016/j.jmb.2011.06.003

Ruberg, F. L., and Berk, J. L. (2012). Transthyretin (TTR) cardiac amyloidosis. Circulation 126, 1286-1300. doi: 10.1161/CIRCULATIONAHA.111.078915

Sant'Anna, R., Gallego, P., Robinson, L. Z., Pereira-Henriques, A., Ferreira, N., Pinheiro, F., et al. (2016). Repositioning tolcapone as a potent inhibitor of transthyretin amyloidogenesis and associated cellular toxicity. Nat. Commun. 7:10787. doi: 10.1038/ncomms10787

Satyal, S. H., Schmidt, E., Kitagawa, K., Sondheimer, N., Lindquist, S., Kramer, J. M., et al. (2000). Polyglutamine aggregates alter protein folding homeostasis in Caenorhabditis elegans. Proc. Natl. Acad. Sci. U.S.A. 97, 5750-5755. doi: 10.1073/pnas.100107297

Saxena, S., and Caroni, P. (2011). Selective neuronal vulnerability in neurodegenerative diseases: from stressor thresholds to degeneration. Neuron 71, 35-48. doi: 10.1016/j.neuron.2011.06.031

Schweizer, R. S., Aponte, R. A., Zimmermann, S., Weber, A., and Reinstein, J. (2011). Fine tuning of a biological machine: DnaK gains improved chaperone activity by altered allosteric communication and substrate binding. Chembiochem 12, 1559-1573. doi: 10.1002/cbic.201000786

Sevigny, J., Chiao, P., Bussiere, T., Weinreb, P. H., Williams, L., Maier, M., et al. (2016). The antibody aducanumab reduces Abeta plaques in Alzheimer's disease. Nature 537, 50-56. doi: 10.1038/nature19323

Shorter, J. (2008). Hsp104: a weapon to combat diverse neurodegenerative disorders. Neurosignals 16, 63-74. doi: 10.1159/000109760

Shorter, J. (2011). The mammalian disaggregase machinery: Hsp110 synergizes with Hsp70 and Hsp40 to catalyze protein disaggregation and reactivation in a cell-free system. PLoS ONE 6:e26319. doi: 10.1371/journal.pone.0026319

Shorter, J. (2016). Engineering therapeutic protein disaggregases. Mol. Biol. Cell 27, 1556-1560. doi: 10.1091/mbc.E15-10-0693

Shorter, J., and Lindquist, S. (2004). Hsp104 catalyzes formation and elimination of self-replicating Sup35 prion conformers. Science 304, 1793-1797. doi: 10.1126/science. 1098007

Shorter, J., and Lindquist, S. (2005). Prions as adaptive conduits of memory and inheritance. Nat. Rev. Genet. 6, 435-450. doi: 10.1038/nrg1616

Shorter, J., and Lindquist, S. (2006). Destruction or potentiation of different prions catalyzed by similar Hsp104 remodeling activities. Mol. Cell 23, 425-438. doi: 10.1016/j.molcel.2006.05.042

Spillantini, M. G., Schmidt, M. L., Lee, V. M., Trojanowski, J. Q., Jakes, R., and Goedert, M. (1997). Alpha-synuclein in Lewy bodies. Nature 388, 839-840. doi: $10.1038 / 42166$

Sun, Z., Diaz, Z., Fang, X., Hart, M. P., Chesi, A., Shorter, J., et al. (2011). Molecular determinants and genetic modifiers of aggregation and toxicity for the ALS disease protein FUS/TLS. PLoS Biol. 9:e1000614 doi: 10.1371/journal.pbio.1000614

Sweeny, E. A., and Shorter, J. (2008). Prion proteostasis: Hsp104 meets its supporting cast. Prion 2, 135-140. doi: 10.4161/pri.2.4.7952

Sweeny, E. A., and Shorter, J. (2016). Mechanistic and structural insights into the prion-disaggregase activity of Hsp104. J. Mol. Biol. 428(9 Pt B), 1870-1885. doi: 10.1016/j.jmb.2015.11.016

Sweeny, E. A., Jackrel, M. E., Go, M. S., Sochor, M. A., Razzo, B. M., DeSantis, M. E., et al. (2015). The Hsp104 N-terminal domain enables disaggregase plasticity and potentiation. Mol. Cell 57, 836-849. doi: 10.1016/j.molcel.2014.12.021

Torrente, M. P., and Shorter, J. (2013). The metazoan protein disaggregase and amyloid depolymerase system: Hsp110, Hsp70, Hsp40, and small heat shock proteins. Prion 7, 457-463. doi: 10.4161/pri.27531

Vacher, C., Garcia-Oroz, L., and Rubinsztein, D. C. (2005). Overexpression of yeast hsp104 reduces polyglutamine aggregation and prolongs survival of a transgenic mouse model of Huntington's disease. Hum. Mol. Genet. 14, 3425-3433. doi: 10.1093/hmg/ddi372

Wallace, E. W., Kear-Scott, J. L., Pilipenko, E. V., Schwartz, M. H., Laskowski, P. R., Rojek, A. E., et al. (2015). Reversible, specific, active aggregates of endogenous proteins assemble upon heat stress. Cell 162, 1286-1298. doi: 10.1016/j.cell.2015.08.041

Wang, A. M., Miyata, Y., Klinedinst, S., Peng, H. M., Chua, J. P., Komiyama, T., et al. (2013). Activation of Hsp70 reduces neurotoxicity by promoting polyglutamine protein degradation. Nat. Chem. Biol. 9, 112-118. doi: $10.1038 /$ nchembio. 1140

Warrick, J. M., Chan, H. Y., Gray-Board, G. L., Chai, Y., Paulson, H. L., and Bonini, N. M. (1999). Suppression of polyglutamine-mediated neurodegeneration in Drosophila by the molecular chaperone HSP70. Nat. Genet. 23, 425-428. doi: $10.1038 / 70532$

Warrick, J. M., Paulson, H. L., Gray-Board, G. L., Bui, Q. T., Fischbeck, K. H., Pittman, R. N., et al. (1998). Expanded polyglutamine protein forms nuclear inclusions and causes neural degeneration in Drosophila. Cell 93, 939-949. doi: 10.1016/S0092-8674(00)81200-3

Winklhofer, K. F., Tatzelt, J., and Haass, C. (2008). The two faces of protein misfolding: gain- and loss-of-function in neurodegenerative diseases. EMBO J. 27, 336-349. doi: 10.1038/sj.emboj.7601930

Yamamoto, A., Lucas, J. J., and Hen, R. (2000). Reversal of neuropathology and motor dysfunction in a conditional model of Huntington's disease. Cell 101, 57-66. doi: 10.1016/S0092-8674(00)80623-6

Yang, C., Wang, H., Qiao, T., Yang, B., Aliaga, L., Qiu, L., et al. (2014). Partial loss of TDP-43 function causes phenotypes of amyotrophic lateral sclerosis. Proc. Natl. Acad. Sci. U.S.A. 111, E1121-E1129. doi: 10.1073/pnas.13226 41111

Yokom, A. L., Gates, S. N., Jackrel, M. E., Mack, K. L., Su, M., Shorter, J., et al. (2016). Spiral architecture of the Hsp104 disaggregase reveals the basis for polypeptide translocation. Nat. Struct. Mol. Biol. 23, 830-837. doi: $10.1038 / \mathrm{nsmb} .3277$

Zhai, R. G., Zhang, F., Hiesinger, P. R., Cao, Y., Haueter, C. M., and Bellen, H. J. (2008). NAD synthase NMNAT acts as a chaperone to protect against neurodegeneration. Nature 452, 887-891. doi: 10.1038/nature06721

Conflict of Interest Statement: The authors declare that the research was conducted in the absence of any commercial or financial relationships that could be construed as a potential conflict of interest.

Copyright (c) 2017 Jackrel and Shorter. This is an open-access article distributed under the terms of the Creative Commons Attribution License (CC BY). The use, distribution or reproduction in other forums is permitted, provided the original author(s) or licensor are credited and that the original publication in this journal is cited, in accordance with accepted academic practice. No use, distribution or reproduction is permitted which does not comply with these terms. 